\title{
Developmental Aspects of Renal $\beta$-Amino Acid Transport. VI. The Role of Membrane Fluidity and Phospholipid Composition in the Renal Adaptive Response in Nursing Animals
}

\author{
RUSSELL W. CHESNEY, NAOMI GUSOWSKI, AND ISRAEL ZELIKOVIC
}

Pediatric Renal Disease Laboratory, Department of Pediatrics, The University of Wisconsin, Madison, Madison, Wisconsin 53792 and The University of California, Davis, School of Medicine, Davis, California 95616

\begin{abstract}
Accumulation of the $\beta$-amino acid taurine is higher in adult rat renal brush border membrane vesicles than in nursing animals, which relates to a higher initial rate Vmax. A low sulfur amino acid diet increases and a $3 \%$ taurine diet reduces the $\mathrm{Vmax}$ of $\mathrm{Na}^{+}$-taurine cotransport in brush border membrane vesicles at all ages after 7 days as compared to values on a normal diet. To determine if changes in membrane fluidity account for these developmental and adaptive events, the fluorescent probe 1,6diphenyl-1,3,5-hexatriene was used to measure fluorescence anisotropy. A two-component, single break curve fit the data over the range 4 to $56^{\circ} \mathrm{C}$. Values for the break temperature centered around $23^{\circ} \mathrm{C}$, not different than a break temperature determined in adult membranes. The values for membrane polarization range from 0.311 to 0.329 in membranes from 7-, 14-, and 21-day-old pups exposed to each of the three diets, significantly lower than the value in adult membranes $(p<0.02)$. The slopes of each component, equivalent to the apparent energy of activation, did not differ in relation to diet. The ontogenic changes in taurine uptake by brush border membrane vesicles related to the exposure to different dietary sulfur amino acid levels are not related to changes in membrane fluidity using 1,6-diphenyl-1,3,5-hexatriene as a probe. However, there is a decrease in fluidity with age. Total phospholipid content falls postweaning, and the percent of total content of phosphatidyl choline and glycerol phosphate fall, and phosphatidyl serine and ethanolamine rise as the rat ages. The results indicate that alterations in the phospholipid composition occur during the process of maturation and that changes in the fluidity of brush border membranes may account for maturational differences in $\mathrm{Na}^{+}$-dependent taurine transport. (Pediatr Res 22: 163167,1987 )
\end{abstract}

\section{Abbreviations}

LTD, low sulfur amino acid diet

NTD, normal sulfur amino acid diet

HTD, high taurine diet

BBMV, brush border membrane vesicles

DPH, 1,6-diphenyl-1,3,5-hexatriene

TMA-DPH, 1-(4-trimethylammoniumphenyl)-6-phenyl1,3,5-hexatriene

Received September 22, 1986; accepted February 27, 1987.

Correspondence and reprint requests Russell W. Chesney, M.D., University of California, Davis Medical Center, Department of Pediatrics, 4301 X Street, Sacramento, CA 95817.

Supported in part from NIH Grants AM31682-02 and AM37223-01. I.Z. is the recipient of a National Kidney Foundation Fellowship.
tPnA, transparinaric acid

BSA, bovine serum albumin

$P$, polarization

PC, phosphatidyl choline

SM, sphingomyelin

Lyso PC, lysophosphatidyl choline

PI, phosphatidyl inositol

PS, phosphatidyl serine

PE, phosphadidyl ethanolamine

PG, phosphatidyl glycerol

ANOVA, analysis of variance

Immature animals of virtually all mammalian species have a "physiologic" generalized aminoaciduria which results in excretion of a higher fraction of the filtered amino acid load of all amino acid classes $(1,2)$. Our laboratory has focused on physiologic factors that modify the development of amino acid transport systems in order to better understand the processes by which these transport sites achieve a mature transport profile (3-7). For an appropriate transport probe, we have used the $\beta$-amino acid taurine, since it is relevant in the nutrition of young animals (8) and since one can manipulate the dietary level of sulfur amino acids to alter the renal handling of taurine $(5,9-14)$. The $\mathrm{Na}^{+}$. dependent accumulation of taurine by BBMV isolated from nursing animals shows enhanced uptake in BBMV prepared from pups whose mothers are fed a LTD and reduced in membranes from pups fed a HTD when compared to membranes from pups whose dams were fed a NTD (15). Taurinuria is greater in nursing or weanling pups as compared to adult controls, but animals of all ages $(7,14,21,28$ day and adult) respond to exposure to the LTD by urinary conservation of taurine and to the HTD by hyperexcretion of this $\beta$-amino acid $(5,15)$.

A precession of taurine uptake occurs, 7-day-old pup membranes $<14$ day $<21$ day $<28$ day $=$ adult. The initial rate of $\mathrm{Na}^{+}$-taurine co-transport $\left(\mathrm{Na}^{+}\right.$-taurine symport) increases with age $(5,15)$. Kinetic analyses of concentration-dependent uptake indicate that this maturational process involves a change in the Vmax of initial $\mathrm{Na}^{+}$-taurine symport with an increase in Vmax by $50 \%$ in LTD fed or exposed pups and a decline after HTD exposure. The factors governing this adaptive change as well as the maturational rise in initial rate are uncertain and could involve membrane-related events. It is clear that alterations in the physical status or in the lipid content of plasma membranes can change the transfer of ions and organic solutes across these membranes (16). The method of fluorescence polarization has been used to describe the "fluidity" of various plasma membranes including those present in the kidney (17). The status of the lipid 
microenvironment of the membrane is also potentially important since the activity of membrane-associated enzymes and transport systems can be modulated by changes in phospholipid composition (18-20). Lipid-protein interactions may also influence the activity of proteins inserted into the membranes (20-22). One can speculate that the renal adaptive response to an altered sulfur amino acid diet may be the result of changes in membrane fluidity and phospholipid composition in conjunction with dietary manipulation.

In this study we examine the influence of three diets varying in their sulfur amino acid composition on membrane fluidity, as measured by the fluorescent probe DPH over a wide range of temperatures. Further, the influence of age on the composition of membrane phospholipids was assessed. These measurements permit one to appreciate if changes in BBMV transport of taurine are governed by alterations in membrane fluidity or phospholipid content.

\section{METHODS}

Animals. Nursing rat pups born to female Sprague-Dawley rats (King Animal Labs, Inc., Oregon, WI) aged 60-90 days were used. The animals were 7,14 , and 21 days of age and were sacrificed after 2 to $3 \mathrm{~h}$ of fasting with access to water only.

Diets. The composition of the diets fed to the nursing mothers is described elsewhere $(9,10)$; maternal rats were placed on the diet at the point of vaginal sperm positivity. Briefly each diet (LTD, NTD, or HTD) is isoproteinic (20\%) and employs soy protein which contains limited amounts of the sulfur-containing $\alpha$-amino acids, methionine and cysteine. The LTD contains $0.2 \%$ methionine, the NTD $0.7 \%$ methionine, and the HTD $0.7 \%$ methionine and $3.0 \%$ taurine $(\mathrm{w} / \mathrm{w})$. The methionine content that permits optional growth is $0.67 \%$ of diet (23). All diets are purchased from ICN, Cleveland, $\mathrm{OH}$.

Preparation of membranes. BBMV are prepared from kidney cortex by a modification of the calcium chloride precipitation method of Booth and Kenny (24) as described previously (9). The method of preparing BBMV from nursing animals has been described previously (15). The final BBMV pellet was resuspended in $290 \mathrm{mM}$ mannitol, $10 \mathrm{mM}$ Tris/HEPES (pH 7.4) and either used fresh or frozen at $-70^{\circ} \mathrm{C}$. Protein was measured according to Lowry et al. (20) using BSA as a standard. In order to assess the purity and relative enrichment of the membranes used in these studies, marker enzyme activities of $\gamma$-glutamyl transferase, $5^{\prime}$-nucleotidase, acid phosphatase (pH 4.8), N-acetyl$\beta$-D-glucosaminidase, succinyl-cytochrome $C$ reductase, malate dehydrogenase, DNA, and ouabain-inhibitable $\mathrm{Na}^{+}+\mathrm{K}^{+}$ATPase were assessed by a previously described technique $(9,10)$. Of all enzymes examined, the final pellet contained an 8- to 12-fold enrichment of $\gamma$-glutamyl transferase and $5^{\prime}$-nucleotidase relative to the initial homogenate and a 0.8 - to 1.2 -fold increase in $\mathrm{Na}^{+}+\mathrm{K}^{+}$ATPase. Enrichment did not vary greatly with age. All other marker enzymes had a lower specific activity indicating excellent enrichment of the brush border fraction.

Fluorescence polarization. In order to determine fluorescence polarization, the lipid soluble fluorescent probe, DPH (Aldrich Chemical Co., Inc., Milwaukee, WI) was used in a $2 \mathrm{mM}$ stock solution in tetrahydrofuran. In a typical study, vesicles $(2-4 \mathrm{mg}$ protein $/ \mathrm{ml}$ ) in $288 \mathrm{mM}$ mannitol, $10 \mathrm{mM}$ Tris/HEPES pH 7.4, and $1 \mathrm{mM} \mathrm{Mg} \mathrm{SO} 4$ were incubated at room temperature with $\mathrm{DPH}$ (final concentration $4 \mu \mathrm{M}$ ) for $15 \mathrm{~min}$. Between four and six separate membrane preparations at each age and exposed to each diet were used to measure fluidity. The extent of $P$ was determined using an Elscint model MV-1 a microviscosimeter (Elscint Inc., Hackensack, NJ). The excitation wavelength was $360 \mathrm{nM}$ and the emission wavelength was $430 \mathrm{~nm}$. This device displays $P=\left(I_{11}-I_{1}\right) \div\left(I_{11}+I_{1}\right)$ where $I_{11}$ and $I_{1}$ represent the fluorescent intensities oriented parallel (11) and perpendicular (1) to the path of polarization of the excitation light (25). Interpretation of the data obtained utilizes the Perrin equation, written $\mathrm{r}^{\circ} / \mathrm{r}=1+3 \tau / \mathrm{P}$, where $\mathrm{r}^{\circ}$ is the maximal limiting anisotropy (0.362) for DPH (26), $\mathrm{r}$ is the anisotropy measured, $\tau$ is the excited state fluorophobe lifetime, and $\mathbf{P}$ is the rotational relaxation time. Fluorescence polarization is generally expressed as the anisotropy parameter $\left[\left(\mathrm{r}^{\circ} / \mathrm{r}\right)-1\right]^{-1}$, where $\mathrm{r}=\left(\mathrm{I}_{11}-\mathrm{I}_{1}\right) \div\left(\mathrm{I}_{11}\right.$ $+2 \mathrm{I}_{1}$ ). Fluorescence polarization was determined from 4 to $56^{\circ}$ $\mathrm{C}$ with readings gathered every $2^{\circ}$ using freshly prepared BBMV. The temperature of the vesicle suspension was directly determined with a digital thermometer (Digitec model 5810) which is accurate to $\pm 0.1^{\circ} \mathrm{C}$.

Concern is warranted over appropriately drawing the shape of the resulting function when $\left[\left(\mathrm{r}^{\circ} / \mathrm{r}\right)-1\right]^{-1}$ is plotted versus reciprocal absolute temperature. Four models were assessed for their ability to fit the data derived: linear, two-phase linear, threephase linear, and quadratic using linear or quadratic regressions where appropriate. The best two- and three-phase linear models were derived using a computer program based on the theory of Hudson (27) as designed by Gourley et al. (28). To test the adequacy of each model, residual plots were determined. $F$ tests were performed to indicate the appropriateness of a two-phase compared to a linear model and if a three-phase model was superior to a two-phase model. Break points and anisotropy parameters were thin compared with results obtained using BBMV from rats of each age and one each diet. Mean values were compared between diets using a two-way analysis of variance.

Phospholipid composition. Phospholipids were extracted and analyzed by established methods (29-31). Frozen membranes from four to six preparations were thawed by incubation at room temperature and a chloroform:methanol $(2: 1, \mathrm{v} / \mathrm{v})$ extract was prepared and washed free of nonlipid contaminants by the method of Raden (30). Phospholipids were isolated by twodimensional thin-layer chromatography using an internal standard consisting of $\left[{ }^{14} \mathrm{C}\right]$ dipalmitoyl $\mathrm{PC}$ added to the membranes to be extracted to measure recoveries for each analysis. Lipid extracts were plated on silica gel $\mathrm{H}$ plates; first direction development was performed in a solvent system of chloroform:methanol/water $(65: 45: 4)$. Plates were then air dired for 10 min, rotated $90^{\circ}$, and developed in tetrahydrofuran: methyl:methanol:water (10:6:4:1). This system allows isolation of PC, SM, Lyso PC, PI, PS, PE, and PG. The method of Bligh and Dyer (29) was used to extract lipids from the gel and phosphorus content was quantitated by the method of Perleman et al. (31). The influence of age on phospholipid composition was compared using Student's $t$ test between groups or ANOVA when indicated.

Supplies. All chemicals used were reagent grade. $\left[{ }^{14} \mathrm{C}\right]$-dipalmitoyl PC and $\left[{ }^{3} \mathrm{H}-\right]$-taurine were purchased from New England Nuclear, Boston, MA. DPH was purchased from Aldrich Chemical Co., Inc., Milwaukee, WI.

\section{RESULTS}

The uptake of taurine ( $\mathrm{Na}^{+}$-dependent component) was greater in BBMV prepared from animals nursed by mothers fed in comparison to animals whose mothers were fed the NTD at 14 and 21 days of age (15). Similarly, the uptake of taurine is diminished in HTD exposed pups at ages 14 and 21 days. At 7 days no difference in uptake is found. These results are presented elsewhere (15) but are mentioned since the membranes prepared for these transport studies were also used to measure membrane fluidity and phospholipid composition herein. Thus, the membrane samples being evaluated demonstrate directly the renal adaptive response to diet, at least 14,21 , and adult membranes. The uptake of taurine is greater in adult membranes than in 21day-old rat pups, which is greater than in 14-day-old rat membranes, which is greater than in 7-day-old rat membranes.

Fluorescence $\mathrm{P}$ data were examined by constructing Arrhenius plots of the $\log \mathrm{P}$ as a function of reciprocal temperature. Residual plots were then derived by a computer best fit model 
which allows evaluation of the adequacy of the four models being examined. In order for a model to be appropriate using such a system, no distinct pattern to the residuals plotted should exist or they should be random. The residual plots for a linear model were parabolic in shape. The two-phase model showed no pattern to its residual plot, whereas a three-phase (two break point) and quadratic model demonstrated marked residual plots. The twophase linear model also had the lowest sum of squares in all 48 data sets $(7,14,21$, adult membranes on each diet performed in quadruplicate). By $t$ test analysis the two-phase linear model was far superior to a linear or three-phase model.

The transition temperatures or the temperature at which the segmented $\log \mathrm{P}$ versus reciprocal temperature function abruptly changes slope centered around $22-24^{\circ} \mathrm{C}$. At any given age the transition temperature is uninfluenced by dietary manipulation. Further, no difference in temperatures between age groups is evident. Table 1 indicates the absolute values for $P$ and the slopes of the upper and lower segments. It should be remembered that each of these slopes represents the apparent energies of activation of the process being examined $(16,18,19)$. At a given age, changes in dietary exposure do not alter either $\mathrm{P}$ or the apparent energies of activation. When a given diet is compared at the different ages examined, these parameters do not differ. Thus diet and age do not change these computer determined kinetic values using a one break, 2-slope model.

When the mean values for $\mathrm{P}$ are examined at each age, age related changes are noted. These values are determined by pooling all results from the 12 membrane preparations at each age. The mean value for $\mathrm{P}$ in 7-day-old rat membranes is $0.3196 \pm$ 0.0064 , in 14-day-old membranes is $0.3183 \pm 0.0084$, and in 21 day-old membranes is $0.3142 \pm 0.0081$. The value in adult membranes is $0.3347 \pm 0.0071$, significantly higher $(p<0.02$ by ANOVA) than in membranes from nursing pups.

The phospholipid composition of the membranes is displayed in Table 2 and Figure 1. The profile of phospholipids differs significantly between adult and immature membranes. Adult membranes contain significantly less PC and glycerol phosphate than membranes from rats of the three ages examined and contain less Lyso-PC and SM than membranes prepared from 7- and 14-day-old animals, respectively. On the other hand, adult membranes contain more PS and PE than immature membranes. We confined these analyses to membranes prepared from NTD animals since no differences in phospholipid content among dietary groups was evident when adult membranes were

Table 1. Values for fluorescence polarization and apparent energies of activation of both linear components

\begin{tabular}{|c|c|c|c|c|c|c|}
\hline & LTD & NTD & HTD & $\begin{array}{c}\text { LTD } \\
v s \text { NTD }\end{array}$ & $\begin{array}{c}\text { NTD } \\
v s \text { HTD }\end{array}$ & $\begin{array}{c}\text { LTD } \\
v s \text { HTD }\end{array}$ \\
\hline \multirow[t]{2}{*}{7 day $P$} & 0.3298 & 0.3059 & 0.3232 & NS & NS & NS \\
\hline & \pm 0.0043 & \pm 0.0126 & \pm 0.0024 & & & \\
\hline \multirow[t]{2}{*}{ Upper segment } & 341.4 & 313.5 & 316.8 & NS & NS & NS \\
\hline & \pm 6 & \pm 12.7 & \pm 11.2 & & & \\
\hline \multirow[t]{2}{*}{ Lower segment } & 211.3 & 181.9 & 189.6 & NS & NS & NS \\
\hline & \pm 6.3 & \pm 22.6 & \pm 13.3 & & & \\
\hline \multirow[t]{2}{*}{14 day $P$} & 0.3198 & 0.3120 & 0.3232 & NS & NS & NS \\
\hline & \pm 0.0050 & \pm 0.013 & \pm 0.0074 & & & \\
\hline \multirow[t]{2}{*}{ Upper segment } & 334.4 & 329.1 & 350.3 & NS & NS & NS \\
\hline & \pm 4.6 & \pm 12.4 & \pm 16.4 & & & \\
\hline \multirow[t]{2}{*}{ Lower segment } & 198.2 & 205.1 & 207.5 & NS & NS & NS \\
\hline & \pm 7.0 & \pm 8.8 & \pm 1.2 & & & \\
\hline \multirow[t]{2}{*}{21 day $P$} & 0.3165 & 0.3000 & 0.3260 & NS & NS & NS \\
\hline & \pm 0.0072 & \pm 0.0167 & \pm 0.0126 & & & \\
\hline \multirow{2}{*}{ Upper segment } & 328.4 & 311.6 & 326.1 & NS & NS & NS \\
\hline & \pm 2.8 & \pm 9.7 & \pm 1.2 & & & \\
\hline \multirow[t]{2}{*}{ Lower segment } & 192.1 & 175.1 & 209.2 & NS & NS & NS \\
\hline & \pm 3.07 & \pm 17.4 & \pm 30.9 & & & \\
\hline \multirow[t]{2}{*}{ Adult $\mathrm{P}$} & 0.3340 & 0.3341 & 0.3361 & NS & NS & NS \\
\hline & \pm 0.0060 & \pm 0.0081 & \pm 0.0106 & & & \\
\hline \multirow[t]{2}{*}{ Upper segment } & 353.0 & 320.3 & 341.1 & NS & NS & NS \\
\hline & \pm 9.10 & \pm 23.1 & \pm 2.9 & & & \\
\hline \multirow[t]{2}{*}{ Lower segment } & 231.1 & 170.4 & 196.8 & NS & NS & NS \\
\hline & \pm 32.1 & \pm 16 & \pm 12.5 & & & \\
\hline
\end{tabular}

Table 2. Phospholipid composition of brush border membranes*

\begin{tabular}{|c|c|c|c|c|c|c|c|c|}
\hline Age & Diet & Lyso PC & SM & PC & PS & PI & $\mathrm{PE}$ & $\mathrm{GP} \dagger$ \\
\hline \multirow{2}{*}{7 day } & & $15.76 \ddagger$ & 86.77 & $252.97 \S$ & $47.1 \ddagger$ & 5.94 & $35.3 \S$ & $4.89 \|$ \\
\hline & & $\pm 2.43^{\circ}$ & \pm 18.34 & \pm 3.74 & \pm 4.98 & \pm 0.66 & \pm 6.07 & \pm 0.95 \\
\hline \multirow[t]{2}{*}{14 day } & NTD & 10.94 & 99.84 & $228.48 \S$ & $56.38 \S$ & 4.94 & $50.42 \ddagger$ & 5.55 \\
\hline & & \pm 1.06 & \pm 4.53 & \pm 24.46 & \pm 6.00 & \pm 0.65 & \pm 2.42 & \pm 0.28 \\
\hline \multirow[t]{2}{*}{21 day } & NTD & 10.65 & $111.76 \|$ & $213.2 \S$ & $55.25 \S$ & 6.86 & $49.7 \ddagger$ & $5.65 \ddagger$ \\
\hline & & \pm 2.12 & \pm 7.05 & \pm 9.73 & $\pm 7.58^{\circ}$ & \pm 1.82 & \pm 7.08 & $\pm 1.19^{\circ}$ \\
\hline \multirow[t]{2}{*}{ Adult } & & 6.78 & 82.27 & 62.48 & 133.5 & 2.50 & 60.39 & 1.27 \\
\hline & & \pm 2.18 & \pm 2.30 & \pm 12.05 & \pm 11.01 & \pm 1.01 & \pm 0.78 & \pm 0.59 \\
\hline
\end{tabular}

${ }^{*} \mu \mathrm{mol} \mathrm{Pi} / \mathrm{mg}$ brush border membrane; mean $\pm \mathrm{SE}, n=4$.

$\dagger$ Glycerol phosphate.

$\ddagger p<0.05$ versus adult.

$\S p<0.001$ versus adult.

$\| p<0.01$ versus adult. 


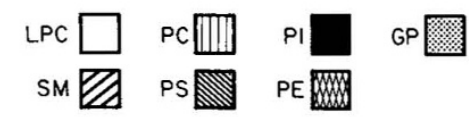
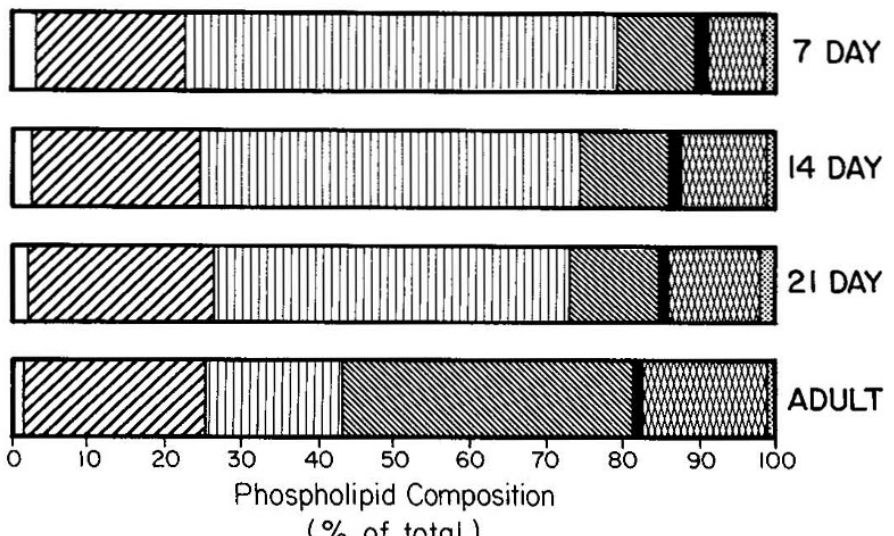

Fig. 1. Phospholipid composition profile expressed as percent of total phospholipid content. Each percent shown is the mean of four determinations. All animals were fed or exposed to the NTD.

examined (Chesney RW, Gusowski N, unpublished observations).

The total amount of phospholipid per mg membrane protein is higher in immature animals membranes: $(448.73 \mu \mathrm{mol} \mathrm{Pi} / \mathrm{mg}$ protein at 7 day; 456.55 at 14 day; 463.07 at 21 day; and 349.18 adult, $p<0.001$ ). The phospholipid content is also expressed as percent of the total phospholipd content (Fig. 1). As can be seen, membranes from immature animals contain from 46 to $56 \%$ PC versus $18 \%$ in adult membranes. PS accounts for $38 \%$ of adult membrane phospholipids in contrast to 10 to $13 \%$ in immature membranes. PE content is also significantly higher in and glycerol phosphate levels are lower in adult membranes.

\section{DISCUSSION}

Because of the large differences in $\mathrm{Na}^{+}$-taurine uptake into vesicles and the difference in initial rate $\mathrm{Na}^{+}$-taurine symport as a result of dietary intervention $(5,15)$, it is appropriate to wonder if changes in the lipid microenvironment of the membrane might underlie these developmental developmental and adaptive changes.

A precedent for this question exists in that numerous studies suggest that changes in the exposure to various nutrients can change both the fluidity and/or the lipid composition of plasma membranes. Further, these changes can influence transport. Changes in salt concentration gradient can alter the degree of lipid peroxidation in porcine intestinal brush border membranes (32), which alters membrane bound enzyme activity. Vitamin D administration is associated with changes in phospholipid composition and with the nature of the fatty acids found in these phospholipids in brush border membrane of the small intestine $(33,34)$. This effect occurs despite inhibition of protein synthesis with cyclohexamide and has been termed a liponomic effect of vitamin $\mathrm{D}$. The initial rate of calcium uptake into BBMV from chick intestine is also influenced by acylation of endogenous phospholipids (35). Cholecalciferol is needed to effect the transfer of phosphatidyl groups from liposomes into the brush border membrane (36).

Dietary changes can induce alterations in the lipid microenvironment and in phospholipids in membranes. Dietary phosphate restriction can change both cholesterol and neutral lipid content of brush border membrane in the rat with a simultaneous change in alkaline phosphatase activity and uptake of phosphate by a $\mathrm{Na}^{+}$-dependent process (37). This reduction in dietary phosphate also is associated with a reduction in membrane fluidity (38).
Since the LTD contains limited amounts of methionine and cysteine, changes in the availability of these sulfur amino acids could potentially limit the synthesis of glutathione from cysteine and its other precursors. It is known that cysteine and cystine taken up into renal tubular epithelium will be incorporated into reduced glutathione, even in neonatal and immature animals, and that glutathione synthesis relates to the level of precursor administered (39). Glutathione, in its reduced form, is a major membrane component protecting against membrane lipid peroxidation (40). A decline in the degree of unsaturation of the phospholipid acyl chains will increase membrane fluidity and could lead to phase separations in the lipid domain of the membrane (40). Although a glutathione-oxidizing agent, such as diamide, does not alter brush border taurine accumulation (41), the potential for changes in sulfur amino acid intake to alter phospholipid composition by such a mechanism and, thereby, to increase fluidity is worthy of study.

At all ages, in terms of DPH fluorescence in relation to temperature, the two-phase linear model gave the best fit. Despite the diets to which these nursing pups were exposed, the transition temperature at which the data points abruptly change slope was similar. These transition temperatures did not vary among 7-, 14-, 21-day-old, or adult rat membranes. Indeed for all ages and all diets this transition temperature is $23 \pm 2^{\circ} \mathrm{C}$. Since the determined values for $\mathrm{P}$ and the upper and lower segment apparent energies of activation are also unchanged by diet and by age, these results indicate that alterations in the physical constituency and in the membrane fluidity, as probed by the fluorescent agent DPH, do not appear to be important in the expression of the renal adaptive response in the brush border membrane. We employed a single membrane fluidity probe, $\mathrm{DPH}$, which has been used with a wide variety of biological membranes $(16,18,19,38,39)$. For this reason we must limit our conclusions regarding the influence of diet on membrane fluidity. However, other groups have shown in renal brush border membranes that other probes including tPnA and TMA-DPH often give similar results even though they distribute differently and as a result may inform on different lipid domains $(37,39$, 41). Other probes are more influenced by ions present in the incubation medium than is DPH (32). Hence we have concentrated our efforts on a single probe.

In contrast to diet, an age-related change in fluidity was noted with a significantly higher $\mathrm{P}$ value in adult membranes. This agerelated increase in $\mathrm{P}$, indicative of reduced fluidity, has been noted by others (42-44). In the present study the values for $\mathrm{P}$ at the break point were higher than in the other reports. This may relate to the point that our membranes were isolated by calcium chloride precipitation (15), rather than magnesium chloride precipitation (43) or magnesium chloride followed by free flow electrophoresis (44). However, the values for $\mathrm{P}$ determined at extreme values $\left(5-15^{\circ}\right.$ and $\left.37-56^{\circ}\right)$ were similar in all four studies. Further each study is consistent in that adult membranes have the highest $\mathrm{P}$ values.

Alterations in the physical state and composition of brush border membrane lipids in relation to maturation have been described. During the process of differentiation from a crypt cell to a villus cell in rat small intestine there is a decline in lipid fluidity and crypt cells have a lower ratio of cholesterol to phospholipid, protein to lipid, and saturated fatty acyl chains to unsaturated chains (22). Changes in phospholipid composition, in phospholipid/cholesterol ratio, and in the incremental change in free energy of transfer of rabbit small intestinal brush border occur with maturity (45). An increment in the Vmax of phosphate uptake by BBMV prepared from fetal sheep kidney as compared to results in the pregnant ewe has been ascribed to a change in membrane lipid fluidity (46). In this study both DPH and DL-2 (9-anthroyl) stearic acid fluorescence increased with age, as well as membrane protein/lipid ratio. The data in this study were consistent with the hypothesis that membrane maturity is characterized by an increase in lipid fluidity. Our failure to show any significant alteration in DPH fluorescence may 
relate to the fact that we are using membranes prepared from postnatal rather than fetal cortex or that our studies are being carried out in rat rather than ovine kidney. Moreover, the change in DPH fluorescence polarization in fetal versus ewe membranes was $0.099 \mathrm{U}$ which is roughly the difference between NTD 7 day-old in comparison to values found in membranes from 14and 21-day-old and adult animals. In our experiments these differences were not significant while they reached $p<0.05$ significance in the ovine studies (45). Further changes between immature and adult membranes were found.

We now have focused on changes in the phospholipid composition of membranes from pups fed or exposed to the NTD, since the increase in the uptake of taurine with age occurs to an equal extent within each dietary group. These studies do indicate that minor but significant differences in phospholipid content are evident with maturity.

Phospholipid composition is influenced by age in that the adult profile in phospholipids found in the membrane is completely different than that found in immature animal membranes. PC and glycerol phosphate are higher and PS and PE are lower in immature membranes, whereas Lyso PC, SM, and PI were unchanged. Whether these changes in the phospholipid profile or the fall in total phospholipid content with aging is related to the higher initial rate uptake of taurine in older rat BBMV is uncertain. Clearly the ratio of protein/phospholipid is higher in membranes from mature animals which is compatible with the notion that more transport sites are inserted into the membrane as part of the maturation process.

In conclusion, $\mathrm{Na}^{+}$-dependent uptake increases with age in isolated BBMV (15). This increase is paralleled by a change in phospholipid composition and by a fall in total phospholipid content, and a change in fluorescence anisotrophy but no change in apparent energies of activation. Thus changes in membrane fluidity in proceeding from immaturity to adulthood may govern these increases in transport. However, the change in taurine transport brought about by diet are not accompanied by differences in DPH fluorescence.

\section{REFERENCES}

1. Segal S 1982 Regulatory aspects of transport during development. In: Spitzer A (ed) The Kidney during Development: Morphology and Function. Masson Publishing, New York, pp 363-375

2. Baerlocher KE, Scriver CR, Mohyuddin F 1971 The ontogeny of amino acid transport in rat kidney. II. Kinetics of uptake and effect of anoxia. Biochim Biophys Acta 249:364-371

3. Chesney RW, Jax DK 1979 Developmental aspects of renal $\beta$-amino acid transport. I. Ontogeny of taurine reabsorption and accumulation in rat renal cortex. Pediatr Res 13:854-860

4. Chesney RW, Jax DK 1979 Developmental aspects of renal $\beta$-amino acid transport. II. Ontogeny of uptake and efflux processes and effect of anoxia. Pediatr Res 13:861-867

5. Chesney RW, Gusowski N, Theissen M 1984 Developmental aspects of renal $\beta$-amino acid transport. IV. Brush border membrane response to altered intake of sulfur amino acids. Pediatr Res 18:611-618

6. Chesney RW, Gusowski N, Friedman AL, Dabbagh S, Diehl A 1985 Divergent membrane maturation in rat kidney: Exposure by dietary taurine manipulation. Int J Pediatr Nephrol 6:93-100

7. Friedman AL, Albright PW, Gusowski N, Padilla M, Chesney RW 1983 Renal adaptation to alteration in dietary amino acid intake. Am $\mathrm{J}$ Physiol 245:F159-F166

8. Chesney RW 1985 Taurine: biological role and clinical implications. Adv Pediatr 32:1-42

9. Chesney RW, Friedman AL, Albright PW, Gusowski N 1982 Fasting reverses the renal adaptation to altered dietary amino intake. Proc Soc Exp Biol Med 170:493-499

10. Chesney RW, Gusowski N, Friedman AL 1983 Renal adaptation to altered dietary sulfur amino acid intake occurs at the luminal brush border membrane. Kidney Int 24:588-594

11. Chesney RW, Gusowski N, Dabbagh S 1985 Renal cortex taurine content regulates renal adaptive response to altered dietary intake of sulfur amino acids. J Clin Invest 76:2213-2221

12. Friedman AL, Albright PW, Chesney RW 1981 Dietary adaptation of taurine transport by rat renal epithelium. Life Sci 29:2415-2419

13. Rozen R, Tenenhouse HS, Scriver CR 1979 Taurine transport in renal brush border membrane vesicles. Biochem J 180:245-248

14. Rozen R, Scriver CR 1982 Renal transport of taurine adapts to perturbed taurine homeostasis. Proc Natl Acad Sci USA 79:2101-2105

15. Chesney RW, Gusowski N, Zelikovic I, Padilla M 1986 Developmental aspects of renal $\beta$-amino acid transport. V. Brush border membrane transport in nursing animals-effects of age and diet. Pediatr Res 20:890-894

16. Jain MK 1980 Phase properties of bilayers. In: Jain MK, Wagner RC (eds) Introduction to Biological Membranes, New York, Wiley, pp 53-175

17. Molitoris BA, Simon FR 1985 Renal cortical brush border and basolatera membranes: cholesterol and phospholipid composition and relative turnover. J Membr Biol 83:207-215

18. Lee AG 1977 Lipid phase transitions and phase diagrams. I. Lipid phase transitions. Biochim Biophys Acta 472:227-281

19. Lee AG 1977 Lipid phase transitions and phase diagrams. II. Mixtures involv ing lipids. Biochim Biophys Acta 472:285-344

20. Ohyashiki T, Taka M, Mohri T 1985 The effects of ionic strength on the protein conformation and the fluidity of porcine intestinal brush border membranes. J Biol Chem 260:6857-6861

21. Brasitus TA, Schacter D, Mamouneas TG 1979 Functional interactions of lipids and proteins in rat intestinal microvillus membranes. Biochemistry 18:4136-4144

22. Brasitus TA and Dudeja PK 1985 Alterations in the physical state and composition of brush border membrane lipids of rat enterocytes during differentiation. Arch Biochim Biophys 240:483-488

23. Rogers AR and Harper AE Jr 1965 Amino acid diets and maximal growth. J Nutr 87:267-273

24. Booth AGE, Kenny AJ 1974 A rapid method for the preparation of microvill from rabbit kidney. Biochem J 142:575-581

25. Bentley KL, Thompson LK, Klebe RJ, Horowitz PM 1985 Fluorescence polarization: a general method for measuring ligand binding and membrane viscosity. Biotechniques 3:356-366

26. Schachter D, Shinitzky M 1977 Fluorescence polarization studies of rat intestinal microvillus membranes. J Clin Invest 59:536-548

27. Hudson DJ 1966 Fitting segmented curves whose joint points have to be estimated. J Am Stat Assoc 61:1097-1129

28. Gourley GR, Mogilevsky W, Odell GB 1983 Hepatic microsomal composition studies in the Gunn Rat. Biochim Biophys Acta 750:419-423

29. Bligh EG, Dyer WJ 1954 A rapid method of total lipid extraction and purification. Can J Biochem Physiol 37:911-917

0. Raden NS 1979 Preparation of lipid extracts. Methods Enzymol 14:245-254

31. Perleman RH, Engle MJ, Kemnitz JW, Kotas RV, Farrell PM 1982 Biochemical and physiological development of fetal rhesus lung. J Appl Physiol 53:230-235

32. Ohyashiki T, Koshino M, Ohta A, Mohri T 1985 The effect of ionic strength on lipid peroxidation of porcine intestinal brush border membrane vesicles. Biochim Biophys Acta 812:84-90

33. Matsumoto T, Fontaine O, Rasmussen H 1981 Effects of 1,25-dihydroxyvitamin $\mathrm{D}_{3}$ on phospholipid metabolism in chick duodenal mucosal cell. J Biol Chem 256:3354-3360

34. Kreutter D, Matsumoto T, Peckham R, Zawalich K, Wen WH, Zolock DT, Rasmussen H 1983 The effect of essential fatty acid deficiency on the stimulation of intestinal calcium transport by 1,25-dihydroxyvitamin $\mathrm{D}_{3}$. J Biol Chem 258:4977-4981

35. Kreutter D, LaFreniere DC, Rasmussen H 1984 The role of fatty acids in the regulation of brush-border $\mathrm{Ca}^{2+}$ transport. Biochim Biophys Acta 777:261266

36. Elgavish A, Rifkind J, Sacktor B 1983 In vitro effects of vitamin $D_{3}$ on the phospholipids of isolated renal brush border membranes. J Membrane Biol 72:85-91

37. Molitoris BA, Alfrey AC, Harris RA, Simon FR 1985 Renal apical membrane cholesterol and fluidity in regulation of phosphate transport. Am J Physio 249:F12-F19

38. Molitoris BA, Alfrey AC, Simon FR 1984 The role of altered proximal tubule brush border membrane cholesterol content in the regulation of phosphate transport. Kidney Int 25:150

39. Foreman JW, Meadow MS, Bovee KC, Segal S 1986 Developmental aspects of cystine transport in the dog. Pediatr Res 20:593-598

40. Shinitzky M, Barenholz Y 1978 Fluidity parameters of lipid regions determined by fluorescence polarization. Biochim Biophys Acta 515:367-394

41. Chesney RW, Gusowski N, Albright P 1985 Inhibition of $\beta$-amino acid transport by diamide does not involve the brush border membrane surface. Pediatr. Pharmacol 5:63-72

42. Hise MK, Weinman EJ 1986 Physical properties of the rat renal brush border membrane during growth. Pflugers Arch 406:234-236

43. Schwarz SM, Hostetler B, Ling S, Mone M, Watkins JB 1985 Intestinal membrane lipid composition and fluidity during development in the rat. Am J Physiol 248:G200-G207

44. Medow MS, Segal S 1987 Age related changes in fluidity of rat renal brushborder membrane vesicles. Biochem Biophys Res Commun (in press)

45. Keelan M, Walker K, Thomson ABR 1985 Intestinal morphology, marker enzymes and lipid content of brush border membranes from rabbit jejenum and ileum: effect of aging. Mech Ageing Dev 31:49-68

46. Moore ES, Rufer L, Brasitus TA, Northrup TE 1985 Apical membrane lipid fluidity (LF) influences phosphate (Pi) transport in fetal kidney. Pediatr Res 19:381A 